\title{
The relationship between triglyceride/high-density lipoprotein cholesterol ratio and coronary slow-flow phenomenon
}

Gonul Aciksari ( $\nabla$ drgonulkutlu@hotmail.com )

Istanbul Medeniyet University Faculty of Medicine: Istanbul Medeniyet Universit Goztepe Prof. Dr.

Suleyman Yalcin City Hospital https://orcid.org/0000-0002-8380-3065

Gokhan CETINKAL

TC Saglik Bakanligi Istanbul Sisli Hamidiye Etfal Egitim ve Arastirma Hastanesi

\section{Mehmet KOCAK}

Fatih Sultan Mehmet EAH: Fatih Sultan Mehmet Egitim ve Arastirma Hastanesi

Adem Atici

Istanbul Medeniyet University Goztepe Prof. Dr Suleyman Yalcin City Hopsital

\section{Fatma Betul Celik}

Istanbul Medeniyet University Goztepe Prof. Dr. Suleyman Yalcin City Hospital

\section{Mustafa Caliskan}

Istanbul Medeniyet University Goztepe Prof. Dr. Suleyman Yalcin City Hospital

\section{Research Article}

Keywords: coronary slow-flow phenomenon, triglyceride, high-density lipoprotein cholesterol

Posted Date: April 28th, 2021

DOl: https://doi.org/10.21203/rs.3.rs-398796/v1

License: (c) (i) This work is licensed under a Creative Commons Attribution 4.0 International License.

Read Full License

Version of Record: A version of this preprint was published at The International Journal of Cardiovascular Imaging on August 28th, 2021. See the published version at https://doi.org/10.1007/s10554-021-02387w. 


\section{Abstract}

Purpose: In this study, we aimed to investigate the relationship between high triglyceride (TG)/highdensity lipoprotein cholesterol (HDL-C) ratio and coronary slow-flow phenomenon (CSFP) in patients undergoing elective coronary angiography for suspected coronary artery disease.

Methods: This prospective study included a total of 84 CSFP patients and 83 controls with normal coronary flow, as evidenced by coronary angiography. The Thrombolysis in Myocardial Infarction (TIMI) frame count (TFC) was used to measure the coronary blood flow velocity. The lipid profiles were analyzed and TG/HDL-C ratio were calculated dividing absolute TG levels by absolute HDL-C levels in peripheral blood.

Results: The median TG/HDL-C ratio was higher in the CSFP group than the control group (3.4 [2.6 to 4.9] vs. 2.3 [1.8 to 3], respectively; $p<0.001)$. The multivariate logistic regression analysis revealed that TG/HDL-C ratio was an independent predictor of CSFP (odds ratio [OR]: 1.78, 95\% confidence interval [CI]: 1.59-2.32; $p=0.001$ ) and TG/HDL-C ratio was positively correlated with the TFC in the CSFP group ( $r$ $=0.311, p<0.001)$. The area under the receiver operating characteristic curve of TG/HDL-C for the diagnosis of CSFP was 0.73 ( $95 \% \mathrm{Cl}: 0.65-0.81 ; \mathrm{p}<0.001$ ). If a cut-off value of 2.75 was used, higher levels of TG/HDL-C ratio could predict the presence of CSFP with $72 \%$ sensitivity and $71 \%$ specificity.

Conclusion: Our study results suggest that TG/HDL-C ratio is associated with CSFP and may be a useful biomarker for predicting CSFP and its severity.

\section{Introduction}

The coronary slow-flow phenomenon (CSFP) is a coronary artery disease, characterized by delayed vessel opacification in the absence of obstructive coronary artery disease [1]. It is defined angiographically and its incidence varies from 1 to $7 \%$ among the patients undergoing coronary angiography for suspected coronary artery disease (CAD) [2]. Although the exact mechanisms of CSFP are still unclear, small vessel disease, endothelial dysfunction, diffuse atherosclerosis, microvascular vasomotor dysfunction, and inflammation are implicated in its pathophysiology [3-6]. In addition, CSFP may cause transient myocardial hypoperfusion in patients with both normal corona arteries and angina, thereby, leading to increasing the risk of CAD and worsening of the prognosis [7]. There is no definitive treatment of CSFP and traditional anti-anginal drugs are often used in the treatment. A particular attention has been paid to control hypertension and dyslipidemia in CSFP cases [8]. In some studies, statins are used to regulate cholesterol levels and take vascular inflammation under control [9].

In recent years, intimal thickening in the coronary arteries, diffuse calcification, lumen irregularity, and non-occlusive atheroma plaques have been identified in the majority of cases with CSFP $[3,10,11]$. In the light of recent findings, CSFP should be evaluated as CAD, which was earlier considered a subgroup of cardiac syndrome $X$ [2]. Several studies have suggested that established cardiovascular risk factors may play a role in the pathogenesis of microvascular angina in healthy individuals, result in coronary 
microvascular dysfunction [12]. Dyslipidemia is one of the risk factors of cardiovascular disease (CVD) [13]. It is defined as a disorder of lipoprotein metabolism, rather than elevations in total cholesterol (TC) or low-density lipoprotein cholesterol (LDL-C) alone [14]. Triglyceride (TG), LDL-C, and high-density lipoprotein cholesterol (HDL-C) are important components of the lipid fraction of the human body [14]. However, epidemiological researches have demonstrated that lipid-related ratios such as TC/HDL-C, TG/HDL-C, or LDL-C/HDL-C may be probably better predictors of CVD risk than any other single lipid marker [15-17]. Among these, the TG/HDL-C ratio was first proposed by Gaziano et al. [17] as an atherogenic index. Several studies have shown that TG/HDL-C ratio is a strong predictor of CAD such as myocardial infarction (MI), LDL phenotype $B$, and atherogenic risk $[18,19]$. In addition, the TG/HDL-C ratio appears to be more valuable than any other single lipid marker, since it has an ability to reflect the complex interactions between the lipoprotein metabolism and to better predict plasma atherogenicity [16]. Besides its simplicity and practicality, there is a growing number of evidences supporting the predictive value of TG/HDL-C ratio in cardiovascular events and may be of clinical relevance [20, 21].

In the light of the literature data, we, in the present study, aimed to investigate the relationship between the TG/HDL-C ratio and CSFP in patients undergoing elective coronary angiography for suspected CAD and to identify whether TG/HDL-C ratio was a feasible biomarker in distinguishing CSFP cases from healthy individuals.

\section{Materials And Methods}

\section{Study design and study population}

This single-center, prospective study was conducted at Department of Cardiology of a tertiary care center between October 2017 and February 2020. A total of 84 CSFP patients who were admitted with chest pain and had myocardial ischemia as assessed by myocardial perfusion scintigraphy with positive treadmill test results and age- and sex-matched 83 controls with normal coronary flow were included. The CSFP was confirmed by coronary angiography and the Thrombolysis in Myocardial Infarction (TIMI) frame count (TFC) was used to measure the coronary blood flow velocity. Those undergoing angiography previously and undergoing surgery or mechanical revascularization for significant CAD were excluded from the study. Patients with left ventricular dysfunction (ejection fraction $<50 \%$ ), peripheral artery disease, congenital heart disease, chronic renal or hepatic insufficiency, chronic obstructive pulmonary disease, acute and chronic inflammatory diseases, or autoimmune disorders, and those receiving drugs affecting the lipid metabolism were also excluded. All participants were informed about the nature of the study and a written informed consent was obtained. The study protocol was approved by the institutional Ethics Committee with the Approval No. 0447. The study was conducted in accordance with the principles of the Declaration of Helsinki.

\section{Data collection and definitions}

Baseline demographic and clinical characteristics of all participants including age, sex, body mass index (BMl; calculated as weight in kg divided by height in meters squared), and comorbidities were 
recorded. Hypertension was defined as a systolic blood pressure of $\geq 140 \mathrm{mmHg}$ and diastolic blood pressure of $\geq 90 \mathrm{mmHg}$ or the current use of antihypertensives. Diabetes mellitus was defined as a fasting blood glucose of $\geq 126 \mathrm{mg} / \mathrm{dL}$ in two consecutive measurements or having a history of diabetes and using insulin or oral antidiabetics. Smoking was defined as current daily smoking.

\section{Coronary angiography-based CSFP assessment}

Coronary angiography was percutaneously performed using the Judkins technique. The coronary arteries were visualized at right and left oblique and caudal planes at 30 frames per second (fps). A contrast agent was injected manually during coronary angiography $(6$ to $10 \mathrm{~mL}$ of contrast agent at each position using right and left, and cranial and caudal angulations). Angiographic images were stored in 34 runs in a CD in accordance with the Digital Imaging and Communications in Medicine (DICOM) standards and the flow velocity was measured. All angiographic measurements were carried out by two cardiologists who were blind to the clinical status of the patients. The flow in coronary arteries was measured using the TFC as described by Gibson et al. [22]. The TFC was calculated from the difference between the first and last frames. The first frame was when nearly complete lumen opacification with antegrade filling, while the final frame was accepted when the contrast dye reached distal landmarks. The following distal landmarks were used for the analysis: the distal bifurcation of the left anterior descending artery (LAD) (i.e., the "mustache") (Figure 1a) and the distal bifurcation of the segment with the longest total distance in the left circumflex artery (LCX) and the first branch of the posterolateral artery in the right coronary artery (RCA) (Figure 1b) [22]. As the LAD artery is often longer than the other main coronary arteries, the LAD TFC is usually high. The longer LAD frame counts were corrected dividing by 1.7 to calculate the corrected TFC [22]. The mean TFC was accepted as $36 \pm 2.5$ for LAD, $22 \pm 4.1$ for LCX, and $20.4 \pm 3.1$ for RCA. The corrected cut-off value for the LAD artery was 21.1( \pm 1.5$)$ frames. Any TFC values with more than 2 standard deviations (SDs) were considered CSFP [21] (Figure 2). The mean TFC for each subject was calculated by summing the TFC for LAD, LCX, and RCA and, then, dividing this sum into 3 [22].

\section{Biochemical analyses}

Venous blood samples were drawn from each participant after an overnight (12-h) fasting. All biochemical and hematological parameters including fasting blood glucose, creatinine, urea, uric acid, aspartate aminotransferase (AST), alanine aminotransferase (ALT), TC, TG, HDL-C, and LDL-C were analyzed on the day of sample collection using a commercial autoanalyzer (c8000i; Abbott Diagnostics $\mathrm{GmbH}$, Germany). For samples with a TG level of $<400 \mathrm{mg} / \mathrm{dL}$, the LDL-C was calculated using the Friedewald formula [(LDL-C = TC $-\mathrm{HDL}-\mathrm{C}-(\mathrm{TG} / 5)]$ [23]. Hematological analyses were carried out using the BC-6800 Hematology Analyzer (Shenzhen Mindray Bio-Medical Electronics Co., Ltd., Shenzhen, China). The lipid profiles were analyzed and TG/HDL-C ratio were calculated dividing absolute TG levels by absolute HDL-C levels in peripheral blood.

\section{Statistical analysis}


Statistical analysis was performed using the SPSS version 26.0 software (IBM Corp., Armonk, NY, USA). Continuous data were expressed in mean \pm SD or median (interquartile range [IQR]), while categorical data were expressed in number and percentage. The normality assumption was checked using the Kolmogorov-Smirnov test. One-way analysis of variance (ANOVA) with post-hoc Tukey test, independent samples $t$-test, Kruskal-Wallis and Mann-Whitney $\mathrm{U}$ tests were used to examine independent quantitative variables. The paired samples $t$-test and Wilcoxon test were performed to analyze dependent quantitative variables. Independent qualitative variables were examined using the chi-square test or Fisher's exact test, when the chi-square assumptions were not met. The Pearson or Spearman correlation analysis was carried out to identify the relationship between the continuous variables. The correlation between the TG/HDL-C ratio and TFC was examined using the Spearman correlation coefficient. Backward stepwise univariate and multivariate logistic regression analyses were performed to determine independent predictors of CSFP. The receiver operating characteristic (ROC) curve was used to identify sensitivity and specificity of independent predictors of CSFP. The optimal cut-off value was calculated from the point of maximal sensitivity and specificity, as described by Youden [24]. The DeLong test was used to compare predictive performance of independent predictors of CSFP. A $p$ value of $<0.05$ was considered statistically significant at $95 \%$ confidence interval.

\section{Results}

Of the 84 CSFP patients, 52 were males and 32 were females with a mean age of $54 \pm 8.9$ (range, 39 to 67 ) years. Of the 83 control individuals, 41 were males and 42 were females with a mean age of $55.2 \pm 8.5$ (range, 33 to 68 ) years. There was no statistically significant difference in the demographic characteristics such as age, sex, BMI and risk factors for CAD such as diabetes mellitus, hypertension, or smoking between the two groups ( $p>0.05$ for all). The use of antiplatelets and angiotensin-converting enzyme inhibitors (ACEI)/angiotensin receptor blockers (ARBs) was more frequent in the control group. Baseline demographic and clinical characteristics of both groups are summarized in Table 1.

Laboratory test results of the CSFP and control groups are presented in Table 2. Accordingly, there was no statistically significant difference in the mean fasting blood glucose, urea, AST, ALT, white blood cell (WBC) count, hemoglobin, and platelets between the groups ( $p>0.05$ for all). However, the mean creatinine and uric acid levels were significantly higher in the CSFP group than the control group $(p=0.002$ and $p=0.004$, respectively). The mean HDL-C level was significantly lower in the CSFP patients $(p=0.001)$ with significantly higher TG levels $(p<0.001)$ and TG/HDL-C ratio $(p<0.001)$. On the other hand, there was no statistically significant difference in the TC and LDL-C levels between the groups $(p=0.208$ and $p=0.209$, respectively). The mean corrected TFC for LAD $46 \pm 5$ vs. $20.4 \pm 2.2$, respectively; $p<0.001$ ), for LCX (30.2 \pm 3.4 vs. $19.7 \pm 2.3$, respectively; $\mathrm{p}<0.001)$, and for RCA $(28.3 \pm 4.2$ vs. $19 \pm 2.3$, respectively; $p<0.001)$ were significantly higher in the CSFP group than the control group. In addition, the mean TFC was significantly higher in these patients $(34.8 \pm 3 v s .19 \pm 1.6$, respectively; $p<0.001)$.

Backward stepwise univariate and multivariate logistic regression analyses were performed to determine independent predictors of CSFP. The relation of CSFP with age, sex, diabetes mellitus, smoking, 
creatinine, uric acid, and TG/HDL-C ratio was examined. Given the fact that TG/HDL-C ratio, TG, and HDL$\mathrm{C}$ show a strong correlation with each other, these variables were excluded from the multivariate analysis. The correlation analysis results are shown in Table 3. Accordingly, there was a positive and significant correlation between the TFC and TG/HDL-C ratio and TG levels $(r=0.311, p<0.01 ; r=0.222, \mathrm{p}=0.06$, respectively) and a negative and significant correlation between the TFC and TG/HDL-C ratio and HDL-C levels $(r=0.275, \mathrm{p}=0.001)$ (Figure 3 ). In the multivariate regression analysis, the TG/HDL-C ratio was found to be a significant independent predictor of CSFP (odds ratio [OR]: $1.78,95 \% \mathrm{Cl}: 1.59-2.32 ; \mathrm{p}=0.001$ ) (Table 4). The ROC curve was used to evaluate the ability of TG, HDL-C, and TG/HDL-C ratio to discriminate CSFP and healthy individuals. The ROC curve showed an area under the curve (AUC) value of 0.72 (\%95 Cl: $0.65-0.81 ; p<0.001$ ). The sensitivity and specificity of TG/HDL-C ratio for predicting CSFP were $72 \%$ and $71 \%$, respectively. The AUC value of TG was 0.69 (95\% Cl: $0.61-0.76 ; p<0.001)$ with $64 \%$ sensitivity and $63 \%$ specificity at values above $126.5 \mathrm{mg} / \mathrm{dL}$. The AUC value of HDL-C was 0.66 (95\% Cl: $0.58-0.73 ; p=0.001$ ) with $59 \%$ sensitivity and $60 \%$ specificity at values below $45.5 \mathrm{mg} / \mathrm{dL}$ (Figure 4 ). The TG/HDL-C ratio, TG, and HDL-C were compared in terms of their predictive performance using the DeLong method. The predictive performance of TG/HDL-C ratio was significantly superior to that of $T G$, but it was borderline significant compared to HDL-C, indicating no statistical significance.

\section{Discussion}

In the present study, we investigated the possible relationship between the TG/HDL-C ratio and CSFP in patients undergoing elective coronary angiography. Our study results showed that the mean TG/HDL-C ratio was significantly higher in the CSFP patients than those with normal coronary flow. In addition, the mean TCF was positively and significantly correlated with the TG/HDL-C ratio. The ROC curve analysis showed that the cut-off value of TG/HDL-C ratio was sufficient to discriminate CSFP and non-CSFP individuals with a high sensitivity and specificity.

The CSFP is an angiographic evidence and should be considered as a distinct entity with specific characteristics, pathogenic mechanisms, and specified diagnostic criteria [1, 2]. Although the exact pathophysiology of CSFP is still unclear, endothelial dysfunction and atherosclerosis have been thought to be involved $[3,6]$. Abnormally slow flow in the coronary arteries has been proposed to be an indicator of diffuse atherosclerosis related to the endothelial damage before angiographically visible coronary lesion, indicating an early manifestation involving both the microvascular system and epicardial coronary arteries $[3,11]$. In a study, Avşar et al. [25]. examined the possible link between CSFP and carotid artery intima-media thickness (CIMT) and found the increased CIMT in CSFP patients. In this study, the CIMT and corrected TCF were also significantly correlated, implying that CSFP might be an early marker of both endothelial dysfunction and subclinical atherosclerosis. In another study investigating the epicardial coronary morphology and intracoronary pressure in CSFP patients using intravascular ultrasonography (IVUS) and flow rate measurements, Cin et al. [3] reported diffuse intimal thickening, widespread calcification through the vessel wall, and non-obstructive atheroma. Consistent with these findings, Pekdemir et al. [11] described longitudinally extended massive calcification along the epicardial arteries as evidenced by IVUS examination as the most common finding in CSFP patients. Furthermore, Camsari 
et al. [26] found the CIMT to be associated with IVUS indices in CSFP patients. Taken together, these findings support the notion that CSFP is an indicator of microvascular diseases and diffuse, nonobstructive atherosclerotic disease of the epicardial arteries.

Endothelial dysfunction is an early indicator of atherosclerosis [27]. Endothelial-dependent, flow-mediated dilatation (FMD) of the brachial artery has been shown to reduce in CSFP patients, indicating that CSFP may play a role in the etiology of endothelial dysfunction [5]. In addition, plasma homocysteine and endothelin levels increase in CSFP patients, both which have detrimental effects on endothelial function $[4,10]$. In the light of existing data, CSFP seems to be a form of early diffuse coronary atherosclerosis, predominantly characterized by microvascular endothelial dysfunction.

It has been well established that lipid abnormalities contribute to the development of atherosclerosis [10]. An extensive number of studies have demonstrated that high TC and LDL-C and low HDL-C are strongly associated with ischemic heart disease, stroke, peripheral vascular disease, and atherosclerosis [12]. Elevated LDL-C levels with high TG and low HDL-C have been shown to increase the risk of CAD than elevated LDL-C levels alone $[28,29]$. Review of the literature reveals that different subfractions of the lipid particles are involved in the atherogenesis. Small dense LDL particles are more atherogenic than larger particles [28]. Less dense HDL2 particles are protective, while small dense HDL3 particles are atherogenic [30]. High TG and low HDL-C levels are strongly associated with small dense LDL-C levels $[18,28]$. The TG/HDL-C ratio has been shown to be an indirect indicator of small dense LDL particles and a superior predictor of coronary heart disease than conventional lipid parameters $[15,19]$. This ratio has been also considered an independent predictor of CVD and all-cause mortality [20, 21]. In their study, Keles et al. [31] evaluated whether the TG/HDL-C ratio differed between patients with chronic inflammatory disease and healthy individuals. The authors concluded that increased TG/HDL-C ratio and low FMD values might indicate endothelial dysfunction and a higher risk of atherosclerosis in these patients. Consistent with previous studies showing a link between low HDL-C and high TG levels and CSFP [32-34], we found high TG levels low HDL-C levels in the CSFP group in our study.

Review of the literature reveals only one retrospective study investigating the relationship between proportional serum lipid parameters and CSFP. In this study including 54 CSFP patients and 39 controls, Kalayci et al. [34] found higher TG, TC/HDL-C, TG/HDL-C, LDL-C/HDL-C, and non-HDL-C levels to be associated with CSFP. In addition, there was a weak, but positive correlation between the TG and TG/HDL-C ratio and mean TFC. In our prospective study, we also found a significant correlation of the high TG, TG/HDL-C ratio, and low HDL-C and CSFP. Furthermore, we showed a weak, but positive correlation between the TG levels and mean TFC and a moderate, positive correlation between the TG/HDL-C ratio and mean TFC. These findings suggest that high TG/HDL-C ratio is an independent predictor of CSFP.

In the literature, several studies have shown the link between LDL-C levels and CSFP [35-37]. In a study investigating the clinical and angiographic properties of CSFP, Yilmaz et al. [35] found LDL-C levels to be correlated with CSFP. On the other hand, no significant correlation between these two variables was 
reported in some other studies [36, 37]. Similarly, in our study, the LDL-C levels were comparable between the two groups. Based on these findings, it can be speculated that TG and HDL-C levels are more valuable indicators of CSFP than LDL-C alone and TG/HDL-C ratio shows a higher correlation with the mean TFC, than TG and HDL-C levels.

Nonetheless, there are some limitations to this study. First, it has a single-center design with relatively small sample size. Second, we were unable to evaluate the atherosclerotic alterations in the coronary arteries via sophisticated imaging modalities such as IVUS or optic coherence tomography, precluding the evaluation of coexisting non-obstructive CAD in isolated CSFP patients. Of note, IVUS is not recommended in the routine daily practice for the evaluation of CSFP patients and the diagnosis is usually confirmed based on visible angiographic findings. Third, inflammatory biomarkers such as the high-sensitivity C-reactive protein, interleukin-6, and tumor necrosis factor-alpha were unable to be analyzed. Further large-scale, prospective, randomized-controlled studies are needed to confirm these findings.

\section{Conclusions}

In conclusion, the CSFP is a poorly recognized clinical entity. Our study results suggest that TG/HDL-C ratio is strongly associated with CSFP and is a significant independent predictor of CSFP. Therefore, it may be a useful biomarker for predicting CSFP and its severity. Beyond its predictive value, it may be of value as a therapeutic target owing to its simplicity and as it allows longitudinal follow-up in the clinical setting. Further studies are warranted to gain a better understanding of the pathogenesis of CSFP and the diagnostic and therapeutic value of TG/HDL-C ratio in this group of patients.

\section{Declarations}

\section{Authors' contributions}

Conceptualization: Gonul AÇIKSARI, Adem ATICl, Methodology: Gonul AÇIKSARI, Adem ATICl, Gokhan CETINKAL Formal analysis: Adem ATICl, Gokhan CETINKAL, Mehmet KOCAK Data collection: Fatma Betul CELIK, Adem ATICI Data interpretation: Adem ATICl, Gokhan CETINKAL, Fatma Betul CELiK Writing: Gonul AÇIKSARI, Mustafa CALISKAN, Original draft: Fatma Betul CELiK, Adem ATICI, Mehmet KOCAK Review \& editing: Mustafa CALISKAN. All authors read and approved the final manuscript.

Conflict of interests: The authors declare they have no conflict of interest.

Funding: This study received no specific grant from any funding agency in the public, commercial or notfor-profit sectors.

\section{References}


1. Tambe AA, Demany MA, Zimmerman HA, Mascarenhas E. Angina pectoris and slow flow velocity of dye in coronary arteries - a new angiographic finding. Am Heart J. 1972; 84: 66-71.

2. Saadat M, Masoudkabir F, Afarideh M, Ghodsi S, VasheghaniFarahani A. Discrimination between Obstructive Coronary Artery Disease and Cardiac Syndrome X in women with typical angina and positive exercise test; utility of cardiovascular risk calculators. Medicina (Kaunas). 2019;55(1):12. E12.s.

3. Cin VG, Pekdemir H, Camsar A, Cicek D, Akkus MN, Parmaksyz T, et al. Diffuse intimal thickening of coronary arteries in slow coronary flow. Jpn Heart J. 2003; 44: 907-919.

4. Li Nan, Tian Liuyang, Ren Jiefeng et al. Evaluation of homocysteine in the diagnosis and prognosis of coronary slow flow syndrome. Biomark Med.2019 Dec; 13(17):1439-1446.

5. Sezgin AT, Sigirci A, Barutcu I, Topal E, Sezgin N, Ozdemir R, et al. Vascular endothelial function in patients with slow coronary flow. Coron Artery Dis. 2003; 14: 155-161.

6. Demir B, Caglar IM, Tureli HO, Pirhan O, Aciksari G, Serkan Çifçi, et al. Coronary slow flow phenomenon associated with high serum levels of soluble CD40 ligand and urotensin II:a multimarker approach. Clin Lab. 2014; 60: 1909-20.

7. Fragasso G, Chierchia SL, Arioli F et al. Coronary slow-flow causing transient myocardial hypoperfusion in patients with cardiac syndrome X: long-term clinical and functional prognosis. Int $J$ Cardiol. 2009; 137:137-144.

8. Zhu X, Shen H, Gao F, et al. Clinical Profile and Outcome in Patients with Coronary Slow Flow Phenomenon Cardiol Res Pract. 2019; 9168153. 
9. Caliskan M, Erdogan D, Gullu H, et al. Effects of atorvastatin on coronary flow reserve in patients with slow coronary flow. Clinical Cardiology. 2007;30(9):475-479.

10. Pekdemir H, Polat G, Cin VG, Çamsari A, Cicek D, Akkus MN, Doven O, Katircibası MT, Muslu N. Elevated plasma endothelin-1 levels in coronary sinus during rapid rate atrial pacing in patients with coronary slow flow. Int J Cardiol. 2004; 97(1):35-41.

11. Pekdemir H, Çamsarı A, Parmaksız T, Çiçek D, Katırcıbaşı MT, Akkuş N, Cin VG, Döven O, Özcan IT. Slow coronary flow may be a sign of diffuse atherosclerosis. Contribution of FFR and IVUS. Acta Cardiol. 2004; 59(2):127-33.

12. Alfonso Sestito, Gaetano A. Lanza, Antonio Di Monaco, Priscilla Lamendola, Giulia Careri, Pierpaolo Tarzia, Gaetano Pinnacchio, Irma Battipaglia and Filippo Crea Relation between cardiovascular risk factors and coronary microvascular dysfunction in cardiac syndrome X. J Cardiovasc Med (Hagerstown). 2011;12(5):322-7.

13. Pearson TA Understanding the Impact of Hyperlipidemia Treatment on Medical Expenditures for Cardiovascular Disease. Med Care. 2017; 55(1): 1-3.

14. Jellinger PS, Handelsman Y, Rosenblit PD, Bloomgarden ZT, Fonseca VA, Garber AJ, et al. American Association of Clinical Endocrinologists and American College of Endocrinology Guidelines for Management of Dyslipidemia and Prevention of Cardiovascular Disease-executive Summary Complete Appendix to Guidelines available at http://journals.aace.com. Endocr Pract. 2017; 23(4):479-97.

15. Zhu L, Lu Z, Zhu L, et al. Lipoprotein ratios are better than conventional lipid parameters in predicting coronary heart disease in Chinese Han people. Kardiol Pol. 2015; 73: 931-938.

16. Millan J, Pinto X, Munoz A, Zuniga M, Rubies-Prat J, Pallardo LF, et al. Lipoprotein ratios: Physiological significance and clinical usefulness in cardiovascular prevention. Vasc Health Risk Manag. 2009; 5: 757-65. 
17. Gaziano JM, Hennekens CH, O’Donnell CJ, Breslow JL, Buring JE. Fasting triglycerides, high-density lipoprotein, and risk of myocardial infarction. Circulation. 1997; 96(8):2520-5.

18. J. Jeppesen, H.O. Hein, P. Suadicani, F. Gyntelberg, Relation of high TG-low HDL cholesterol and LDL cholesterol to the incidence of ischemic heart disease. An 8-year follow-up in the Copenhagen Male Study, Arterioscler. Thromb. Vasc. Biol. 1997; 17 (6): 1114-1120.

19. Hanak V, Munoz J, Teague J, Stanley A Jr, Bittner V. Accuracy of the triglyceride to high-density lipoprotein cholesterol ratio for prediction of the low-density lipoprotein phenotype B. Am J Cardiol. 2004; 94(2):219-22.

20. Wan $\mathrm{K}$, Zhao J, Huang $\mathrm{H}$, et al. The association between triglyceride/high-density lipoprotein cholesterol ratio and all-cause mortality in acute coronary syndrome after coronary revascularization. PLoS One. 2015;10: e0123521.

21. Sultani R, Tong DC, Peverelle M, et al. Elevated triglycerides to high-density lipoprotein cholesterol (TG/HDL-C) ratio predicts long-term mortality in high-risk patients. Heart Lung Circ. 2020; 29: 41421.

22. Gibson CM, Cannon CP, Daley WL, Dodge JT, Jr. Alexander B Jr, Marble SJ, McCabe CH, Raymond L, Fortin T, Poole WK, Braunwald E. TIMI frame count: a quantitative method of assessing coronary artery flow. Circulation 1996; 93: 879-88.

23. Friedewald WT, Levy RI, Fredrickson DS. Estimation of the concentration of low-density lipoprotein cholesterol in plasma, without use of the preparative ultracentrifuge. Clin Chem. 1972; 18(6):499-502.

24. Youden WJ. Index for rating diagnostic tests. Cancer. 1950;3(1):32-5. 
25. AvşarO, Demir I, Ekiz $O$ et al. Relationship between the slow coronary flow and carotid artery intimamedia thickness. Anadolu Kardiyol Derg 2007;7(1):19-23.

26. Camsari A, Ozcan T, Ozer C, et al. Carotid artery intima-media thickness correlates with intravascular ultrasound parameters in patients with slow coronary flow. Atherosclerosis 2008; 200:310-4.

27. Davignon J, Ganz P: Role of endothelial dysfunction in atherosclerosis. Circulation 2004; 109, $27-$ 32.

28. Vakkilainen J, Steiner G, Ansquer JC, et al. Relationships between low-density lipoproteinparticle size, plasma lipoproteins, and progression of coronary artery disease: the Diabetes Athero-sclerosis Intervention Study (DAIS). Circulation 2003; 107: 1733-1737.

29. Miller NE. Associations of high-density lipoprotein subclasses and apolipoproteins with ischemic heart disease and coronary atherosclerosis, Am Heart J. 1987;113:589-97.

30. Carmena R, Duriez P, Fruchart JC. Atherogenic lipoprotein particles in atherosclerosis. Circulation 2004; 109: 2-7.

31. Keles $\mathrm{N}$, Aksu F, Aciksari $\mathrm{G}$ et al. Is triglyceride/HDL ratio a reliable screening test for assessment of atherosclerotic risk in patients with chronic inflammatory disease? North Clin Istanbul. 2016; 3(1):39-45.

32. Sanati H, Kiani R, Shakerian F, Firouzi A, Zahedmehr A, Peighambari M, et al. Coronary slow flow phenomenon clinical findings and predictors. Res Cardiovasc Med. 2016;5(1): e30296.

33. Sezgin AT, Barutcu I, Sezgin N, Gullu H, Esen AM, Acikgoz N, et al. Contribution of plasma lipid disturbances to vascular endothelial function in patients with slow coronary flow. Angiology. 2006; 57(6):694-701.

34. Kalaycı B, Kalaycı S, Kokturk F Proportional Serum Lipid Parameters in Coronary Slow Flow Phenomenon Turkiye Klinikleri J Cardiovasc Sci. 2019;31(1):21-8. 
35. Yilmaz H, Demir I, Uyar Z. Clinical and coronary angiographic characteristics of patients with coronary slow flow. Acta Cardiol. 2008; 63(5):579-84.

36. Ding YD, Pei YQ, Wang R, Yang JX, Zhao YX, Liu XL, Shen H, Ma Q, Zhang S, Ge HL Increased plasma lipoprotein-associated phospholipase A2 levels are associated with coronaryslowflow. BMC Cardiovasc Disord. 2020; 20(1): 248.

37. Yazici M, Demircan S, Aksakal E, Sahin M, Meriç M, Dursun I, et al. Plasma insulin, glucose and lipid levels, and their relations with corrected TIMI frame count in patients with slow coronary flow. Anadolu Kardiyol Derg. 2003;3(3):222-6.

\section{Tables}

Table 1. Baseline demographic and clinical characteristics of participants

\begin{tabular}{|c|c|c|c|c|c|c|}
\hline \multirow{3}{*}{$\frac{\text { Variable }}{\text { Age, year }}$} & \multirow{2}{*}{\multicolumn{2}{|c|}{$\begin{array}{c}\text { CSFP group }(\mathrm{n}=\mathbf{8 4}) \\
\text { Mean } \pm \text { SD } / \mathrm{n}(\%)\end{array}$}} & \multirow{2}{*}{\multicolumn{3}{|c|}{$\begin{array}{c}\text { Control group ( } \mathrm{n=83)} \\
\text { Mean } \pm \text { SD/n }(\%)\end{array}$}} & \multirow{3}{*}{$\begin{array}{c}p \text { value } \\
0.646\end{array}$} \\
\hline & & & & & & \\
\hline & 54.6 & \pm 8.9 & 55.2 & \pm & 8.5 & \\
\hline \multirow[t]{2}{*}{ Sex, n (\%) } & 32 & $(38.1)$ & 42 & & $(50.6)$ & 0.104 \\
\hline & 52 & (61.9) & 41 & & $(49.4)$ & \\
\hline $\mathrm{BMI}, \mathrm{kg} / \mathrm{m}^{2}$ & 28.7 & \pm 3.5 & 29.1 & \pm & 3.6 & 0.338 \\
\hline Smoking, n (\%) & 16 & (19) & 21 & & $(25.3)$ & 0.331 \\
\hline Hypertension, n (\%) & 38 & $(45.2)$ & 39 & & $(47)$ & 0.821 \\
\hline Diabetes mellitus, n (\%) & 22 & $(26.2)$ & 19 & & $(22.9)$ & 0.620 \\
\hline Calcium canal blocker, n (\%) & 16 & (19) & 10 & & $(12)$ & 0.212 \\
\hline Beta-blocker, n (\%) & 18 & $(21.4)$ & 26 & & (31.3) & 0.147 \\
\hline ACEI/ARB, n (\%) & 16 & $19,0 \%$ & 27 & & $32,5 \%$ & 0.046 \\
\hline Antiplatelet, n (\%) & 18 & $21,4 \%$ & 30 & & $36,1 \%$ & 0.036 \\
\hline OAD & 15 & $17,9 \%$ & 15 & & $18,1 \%$ & 0.971 \\
\hline Statin & 12 & $14,3 \%$ & 16 & & $19,3 \%$ & 0.388 \\
\hline
\end{tabular}

Data are given in mean $\pm S D$ or number and frequency, unless otherwise stated. ACEI: angiotensin-converting enzyme inhibitor; ARB: angiotensin receptor blocker; BMI: body mass index; CSFP: coronary slow flow phenomenon; NCF normal coronary flow; SD: standard deviation.

Table 2. Laboratory test results of participants 


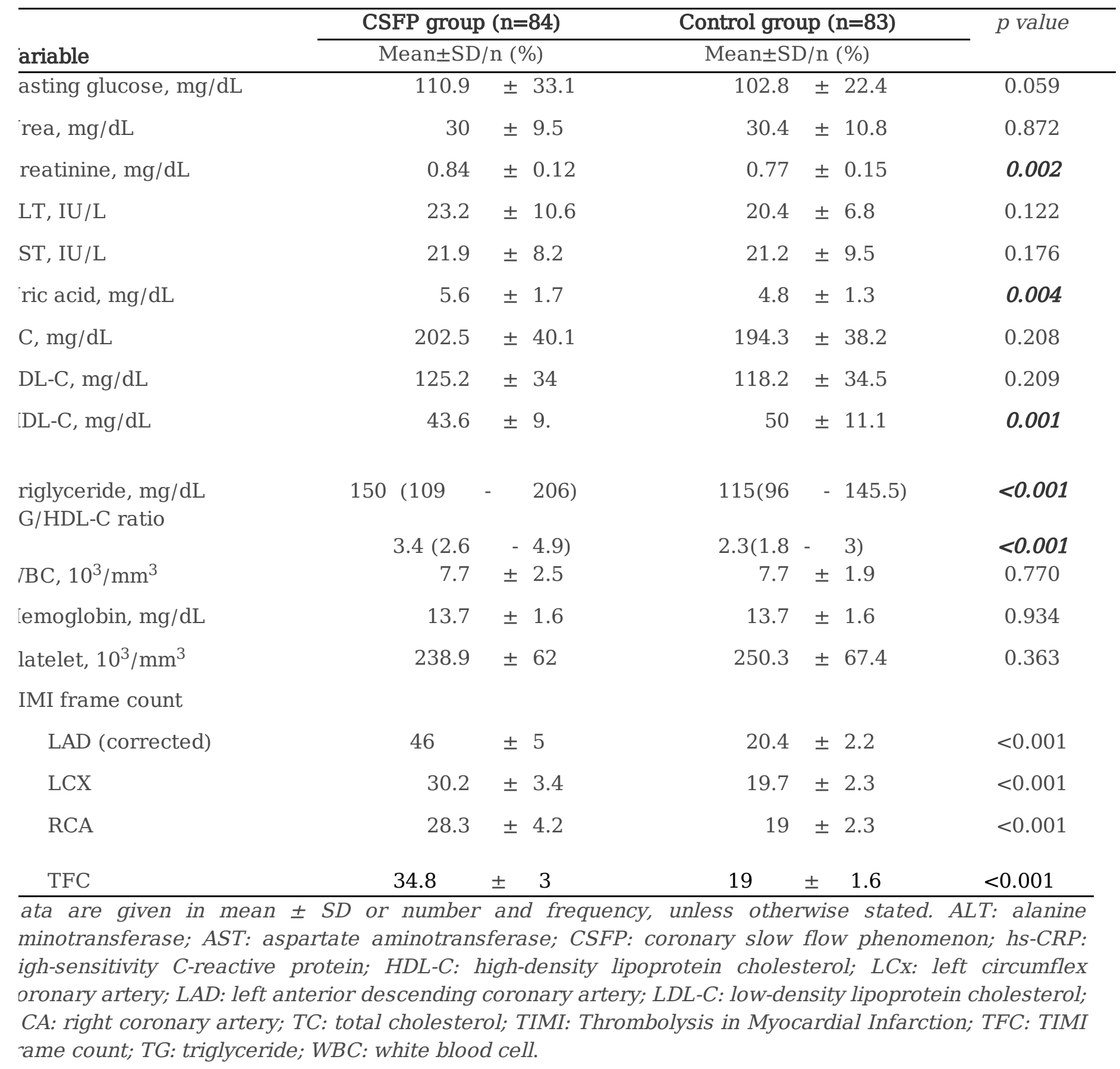

Table 3. Correlation analysis results 


\begin{tabular}{|c|c|c|c|c|c|c|}
\hline \multirow[b]{2}{*}{ Variable } & \multicolumn{3}{|c|}{ Univariate Model } & \multicolumn{3}{|c|}{ Multivariate Model } \\
\hline & OR & $95 \% \mathrm{CI}$ & $p$ & OR & $95 \% \mathrm{CI}$ & $p$ \\
\hline Age & 0.992 & $0.958-1.027$ & 0.644 & & & \\
\hline
\end{tabular}

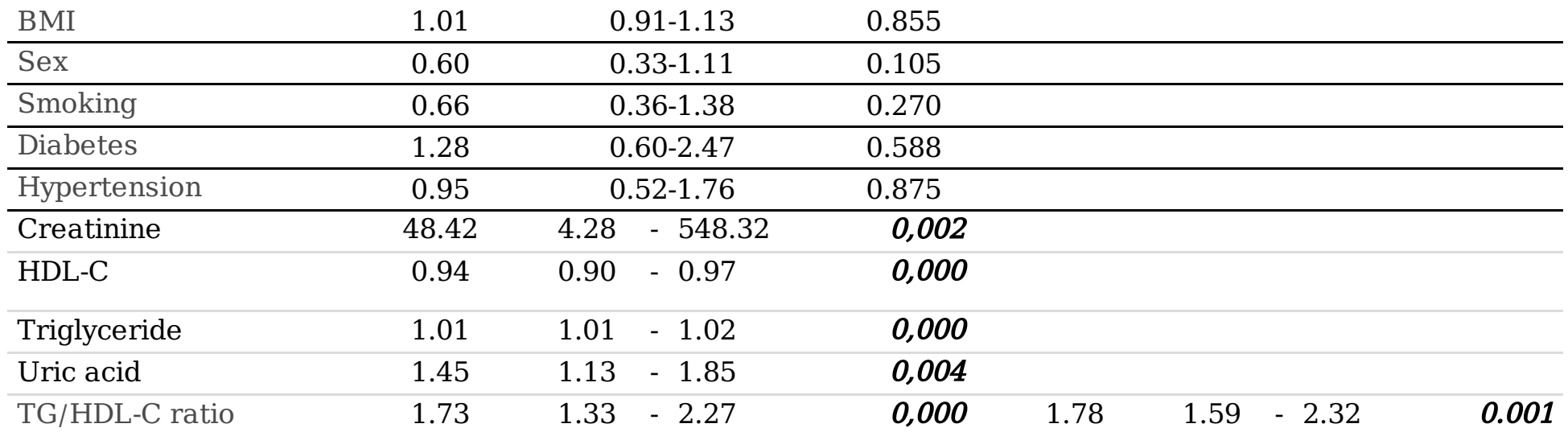

Logistic Regression (backward LR). BMI: body mass index; HDL-C: high-density lipoprotein cholesterol; LDL-C: low-density lipoprotein cholesterol; TG: triglyceride; OR: odds ratio; CI: confidence interval.

Table 4. Correlation of mean TFC with other variables

r $\quad p$ value

$\begin{array}{lcc}\text { Fasting glucose } & 0.115 & 0.154 \\ \text { Creatinine } & 0.112 & 0.157 \\ \text { Urea } & 0.069 & 0.385 \\ \text { Uric acid } & 0.109 & 0.166 \\ \text { TC } & 0.003 & 0.975 \\ \text { TG/HDL-C ratio } & 0.311 & <0.001 \\ \text { LDL-C } & 0.002 & 0.809 \\ \text { HDL-C } & -0.275 & \mathbf{0 . 0 0 1} \\ \text { Triglyceride } & 0.222 & \mathbf{0 . 0 0 6}\end{array}$

HDL-C: high-density lipoprotein cholesterol; LDL-C: low-density lipoprotein cholesterol; TC: total cholesterol; TFC: Thrombolysis in Myocardial Infarction frame count; TG: triglyceride.

\section{Figures}




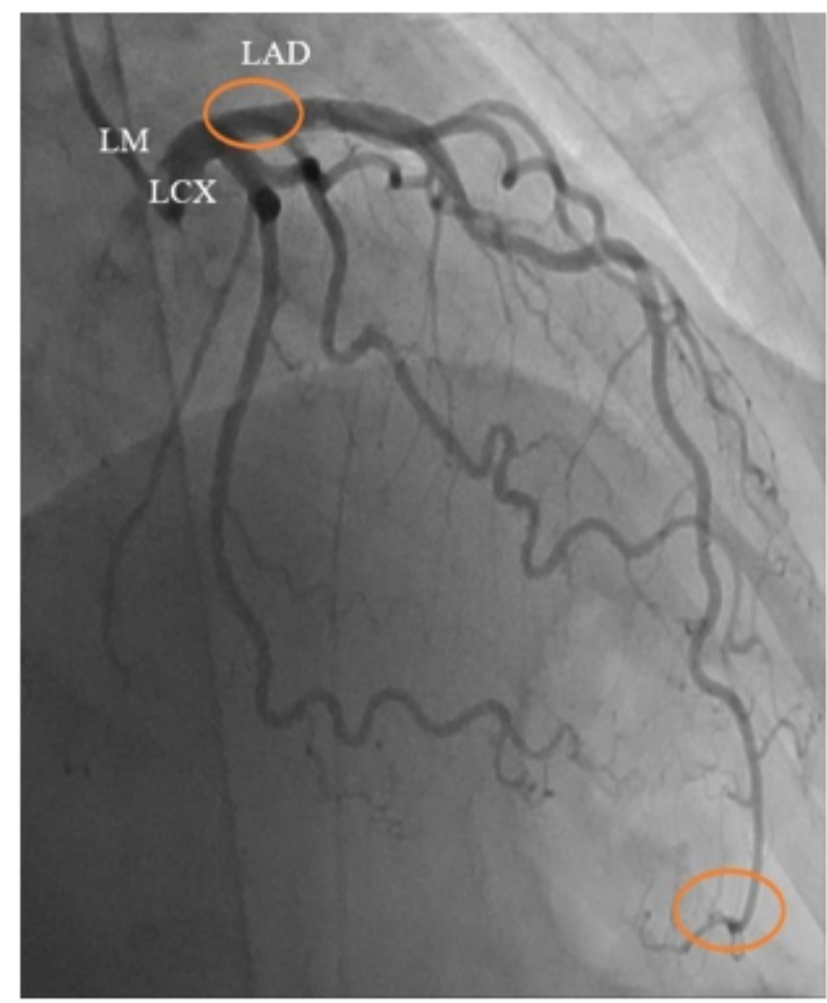

la

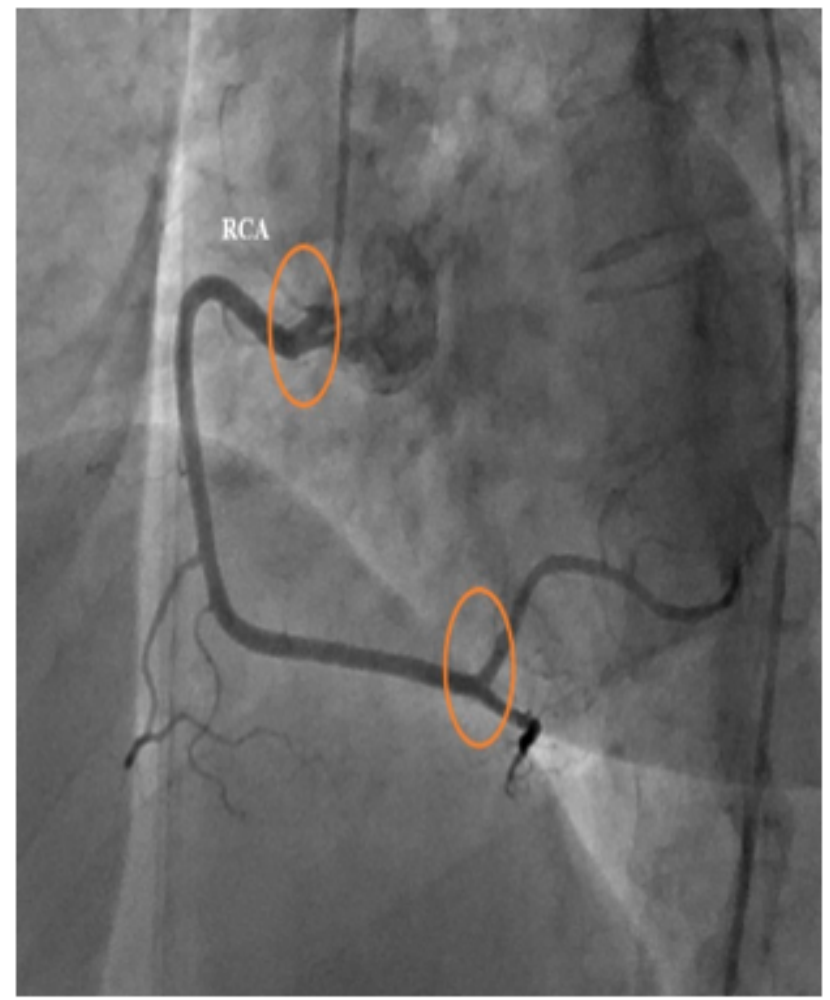

lb

\section{Figure 1}

a: Measurement of coronary flow by TFC. The speed with which the dye reaches the distal 'pitchfork' bifurcation of LAD from the ostium is measured in frame counts. b: In RCA the flow is measured from ostium to origin of first posterolateral branch. *Abbreviations: TFC, Thrombolysis in Myocardial Infarction frame count; $L A D$, left anterior descending; RCA, right coronary artery; LCX, left circumflex; LM, left main.

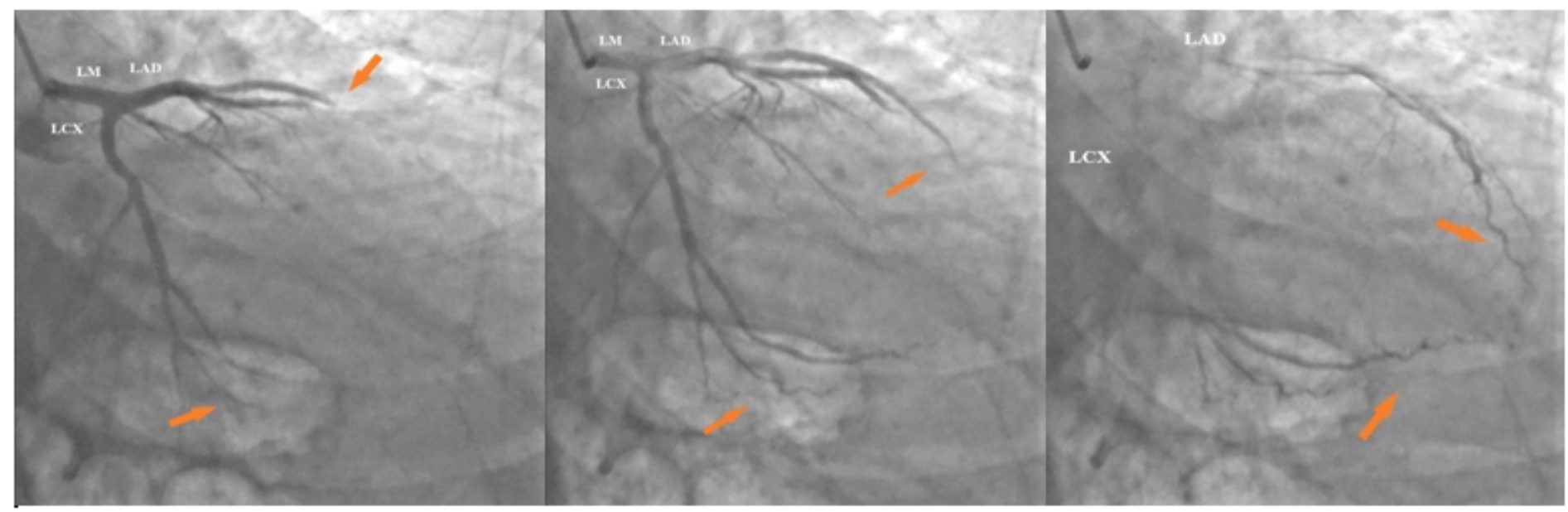

\section{Figure 2}

Coronary angiography of a 46-year-old man with CSFP of our study participant showing slow progression of contrast material In the LAD and with normal epicardial coronary arteries. TIMI frame count 55. 
*Abbreviations: CSFP, coronary slow-flow phenomenon; LAD, left anterior descending; LCX, left circumflex; LM, left main; TIMI, Thrombolysis in Myocardial Infarction.

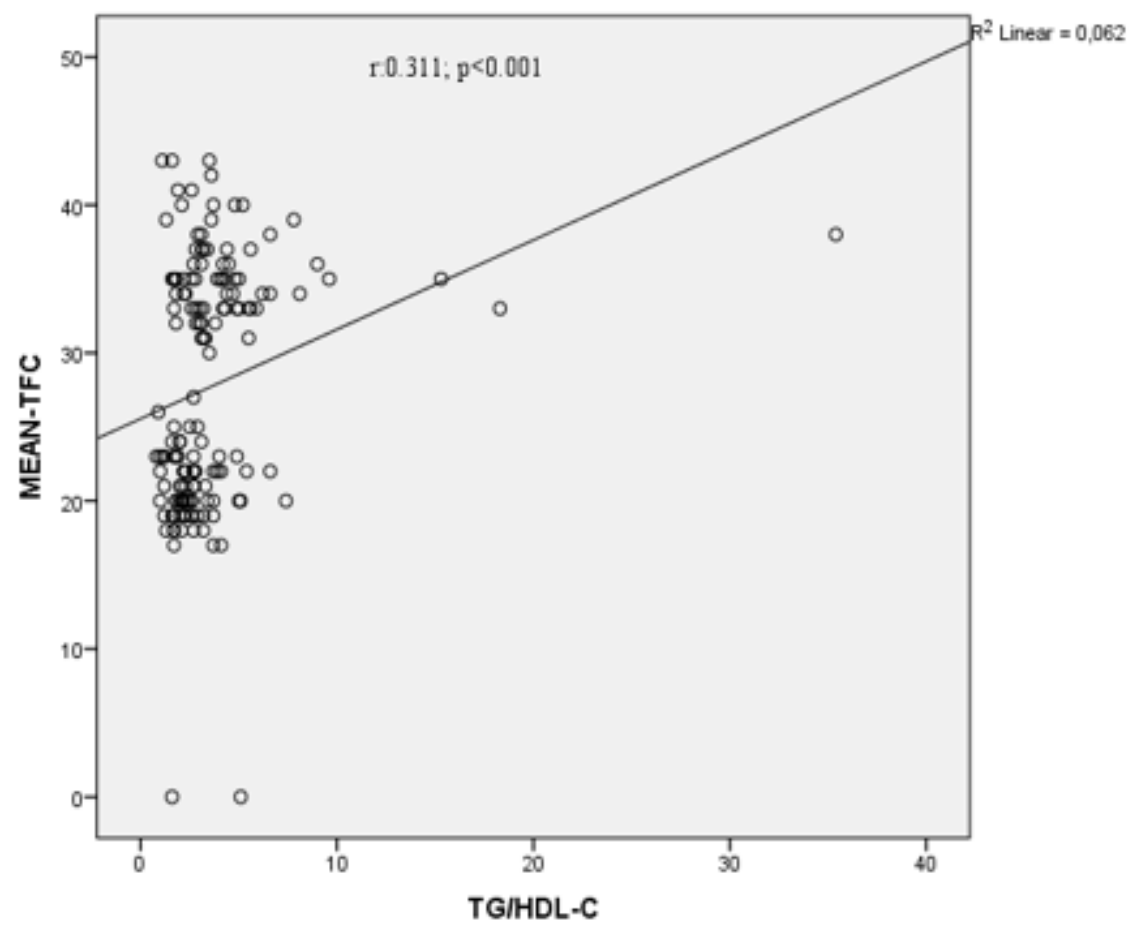

\section{Figure 3}

Correlation between TG/HLD-C ratio and the mean-TFC in CSFP patients. TFC: Thrombolysis in Myocardial Infarction frame count; TG: triglyceride; HDL-C: high-density lipoprotein cholesterol.

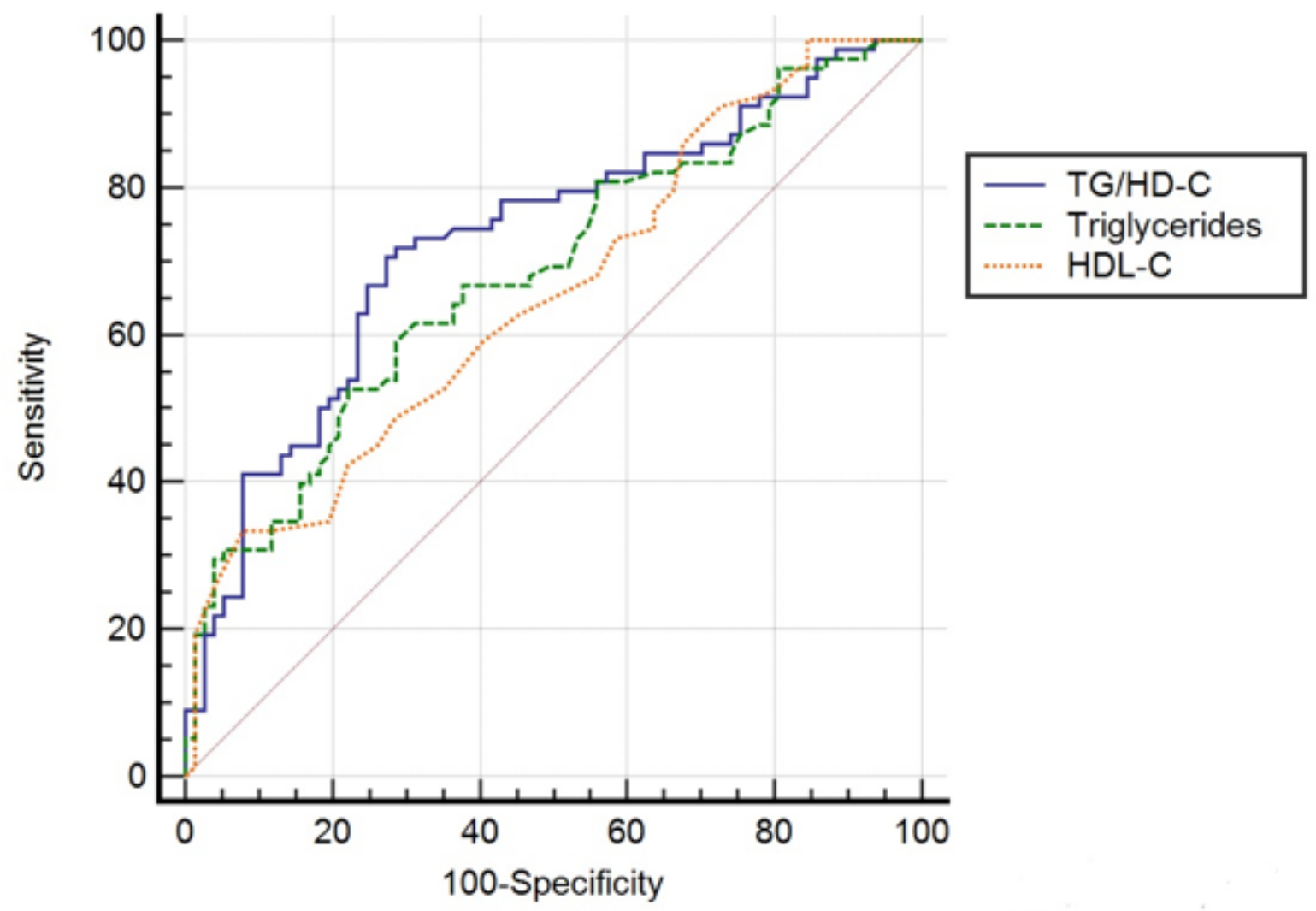


Figure 4

ROC curves showing the predictive value of TG/HDL-C ratio, TG, and HDL-C for CSFP. ROC: receiver operating characteristics; AUC: area under the curve; TG: triglyceride; HDL-C: high-density lipoprotein cholesterol; CSFP: coronary slow flow phenomenon; $\mathrm{Cl}$ : confidence interval. Differences between areas are as follows: TG-HDL-C vs. TG: 0.444; 95\% Cl: 0.037-0.084; Z-score: 2.14; $p=0.03$; TG/HDL-C vs. HDL-C: 0.073; 95\% Cl: -0.0034-0.15; Z-score: 1.87; $p=0.06$; TG vs. HDL-C: 0.029; 95\% Cl: -0.0772-0.14; Z-score: $0.54 ; p=0.59)$. 